\title{
DISTRIBUIÇÃO E ABUNDÂNCIA DO ICTIOPLÂNCTON NA COSTA NORTE DA BAHIA, BRASIL.
}

Recebido: $10 / 03 / 2004$

Aceito: 18/10/04

\author{
PAUlo De Oliveira MAFALda JR. ${ }^{1,2}$ \\ CHOSSI SINQUE ${ }^{2}$ \\ José HENRIQUE MUELBERT ${ }^{2}$ \\ Christiane SAMPAIO DE SOUZA ${ }^{1}$
}

1 - Laboratório de Plâncton, Instituto de Biologia

Universidade Federal da Bahia

Salvador, Brazil 40.210-020

pomafa@ufba.br

2 - Curso de Pós-graduação em Oceanografia

Biológica

Universidade Federal do Rio Grande

Rio Grande, Brazil 96.200-010

\section{RESUMO}

As variações espaciais e sazonais da distribuição e abundância do ictioplâncton foram estudados na costa norte da Bahia. Amostragens foram feitas em 12 estações em maio/1993, setembro/1993, dezembro/1993 e março/1994. O ictioplâncton foi coletado através de arrastos oblíquos utilizando-se rede bongo com malha de $500 \mu \mathrm{m}$. Foram dentificadas 12 Ordens, 33 famílias e 20 espécies de larvas de peixes. A abundância relativa das famílias características foram as seguintes: Gobiidae (25\%), Gerreidae (11\%), Engraulidae (11\%), Clupeidae (10\%), Carangidae (7\%), Myctophidae (5\%), Haemulidae (5\%), e Scombridae (3\%). A densidade de ovos de peixes apresentou coeficientes de correlação de Pearson positivos e significativos ( $\mathrm{r}>0,7$ ), com a temperatura, turbidez, ortofosfato, biomassa fitoplanctônica e biomassa de microplâncton. A densidade de larvas de peixes apresentou coeficientes de correlação positivo e significativos ( $\mathrm{r}$ > 0,7), com a saturação de oxigênio e salinidade. A utilização do litoral norte da Bahia, como sítio de desova e criação de larvas de peixes, foi verificada em todas as épocas do ano, com maior densidade de ovos no período chuvoso (água Costeira) e maior densidade de larvas durante o período seco (água Oceânica).

Palavras chave: Ictioplâncton, Distribuição, Costa Norte da Bahia

\section{Abstract \\ Ichthyoplankton distribution and abundance at Bahia north shore, Brazil.}

The ichthyoplankton spacial and seasonal distribution and abundance were studied at Bahia north shore. Samplings were carried out in 12 stations in: May, September, December of 1993 and March of 1994. The ichthyoplankton was collected through oblique hauls using a bongo net $500 \mu \mathrm{m}$ mesh size. Twelve orders, 33 families and 20 species of fish larvae were identified. The relative abundance of main families was Gobiidae (25\%), Gerreidae (11\%), Engraulidae (11\%), Clupeidae (10\%), Carangidae (7\%), Myctophidae (5\%), Haemulidae (5\%), and Scombridae (3\%). Fish egg 
density presented a significant and positive Pearson coefficient correlation ( $r>0.7$ ) with: temperature, phosphate, turbidity, phytoplankton biomass and microzooplankton biomass. The fish larvae density presented significant and positive Pearson coefficient correlation $(r>0.7)$ with oxygen and salinity. It was observed that the Bahia north coast have been used as a spawning and growning area during all the year, with higher egg density, in the rainy season (Coastal water) and greater larvae density, during the dry season (Oceanic water).

Key words: Ichthyoplankton, Distribution, Bahia north shore.

\section{INTRODUÇÃO}

As regiões costeiras marinhas constituem habitats favoráveis para os estágios iniciais da vida dos peixes que as utilizam como áreas de desova e criação, sendo frequentada por espécies ecologicamente distintas ou que exibem diferentes hábitos de desova (DOYLE et al., 1993; LEIS, 1993). Estes ambientes são caracterizados pela maior disponibilidade de alimento, baixa abundância de predadores (FRANK; LEGGETT, 1983), além de padrões de circulação que favorecem a retenção dos estágios ictioplanctônicos (CASTILLO et al., 1991). Apesar da importância no ciclo de vida dos peixes, é limitado o conhecimento sobre sua utilização por diferentes espécies e os fatores que influenciam a distribuição e abundância dos taxa. Assim, documentar sua utilização em escala espacial e temporal, constitui um passo essencial em direção a compreensão e previsão dos efeitos das mudanças ambientais sobre as populações de peixes (LAPRISE; PEPIN, 1995).

Os primeiros estudos sobre o ictioplâncton costeiro do Nordeste Brasileiro realizados na Baía de Todos os Santos (MAFALDA JR.,1995), e no sistema estuarino-lagunar de Jequiá (MAFALDA JR; SILVA, 1996), apontam a predominância de famílias pelágicas (Engraulidae, Carangidae e Gerreidae), associadas a recifes de corais (Gobiidae) e também demersais (Sciaenidae). No ictioplâncton oceânico presente na Zona Econômica Exclusiva predominam os mesopelágicos (Myctophidae, Gonostomatidae e Paralepididae), as famílias associadas a recifes de corais (Scaridae e Gobiidae), os demersais (Bothidae), além de epipelágicos como os Carangidae, Bramidae e Scombridae (EKAU; WESTHAUS-EKAU, 1996; MAFALDA JR. et al., 1997, 2002b; SILVA, 1997; SOUZA, 1999; EKAU et al., 1999; VELAME, 2001; PINTO et al., 2002). O ictioneuston dos bancos e ilhas oceânicas do nordeste Brasileiro foi caracterizado pela dominância de Exocoetidae e Hemiramphidae (LESSA et al., 1999).

O aporte de nutrientes provenientes dos estuários favorecem um aumento da biomassa primária e secundária permitindo a formação de sítios de desova na região de quebra de plataforma continental da Zona Econômica Exclusica do Nordeste, onde foram encontradas elevadas concentrações de ovos e larvas de peixes (CARROZZO et al., 1999; EKAU; WESTHAUS-EKAU, 1996; EKAU et al., 1999; MAFALDA JR. et al., 2002b).

A área de estudo possui cerca de $580 \mathrm{~km}^{2}$ e apresenta uma plataforma continental estreita, cuja extensão atinge $10 \mathrm{~km}$ na altura dos $13{ }^{\circ} \mathrm{S}$ (BRANDINI et al., 1997). O padrão de precipitação pluviométrica apresenta um período seco, entre setembro e fevereiro e, um período chuvoso entre março e agosto (MAFALDA JR. et al., 2002a; SEI, 1999). Os rios Pojuca e Jacuípe, no setor norte e Joannes, no setor sul, levam a formação de pequenos estuários (PAREDES; FREITAS, 1980; PAREDES et al., 1993), mas o padrão oceanográfico predominante é determinado pela massa de Água Tropical, com salinidade entre 36-38, e temperatura entre $24-26^{\circ} \mathrm{C}$ (GARFIELD, 1990). As correntes que atuam paralelas a linha de costa, no sentido NE-SW, possuem características oligotróficas devido ao escasso teor em nutrientes inorgânicos (PAREDES, 1991).

A pouca informação sobre a composição e ecologia do ictioplâncton da costa norte da Bahia (MAFALDA JR et al., 1999), estimulou o desenvolvimento deste estudo, que possui como objetivos: determinar a ocorrência, distribuição e abundância de ovos e larvas de peixe e, investigar as variações espaciais e sazonais da ictiofauna larval. 


\section{Metodologia}

As posições das estações de amostragem no litoral norte da Bahia, entre Açú da Torre $\left(12^{\circ} 32^{\prime}\right.$ S e $\left.37^{\circ} 57^{\prime} \mathrm{W}\right)$ e Itapuã $\left(12^{\circ} 58^{\prime} \mathrm{S}\right.$ e $\left.38^{\circ} 21^{\prime} \mathrm{W}\right)$, foram obtidas através do navegador por satélite modelo "Garmin GPS 50". As coletas foram realizadas com lancha de pequeno porte, em 12 estações de amostragem, nos meses de maio/1993, setembro/1993, dezembro/1993 e março/1994 (Fig.1). Os dados meteorológicos foram fornecidos pelo Instituto Nacional de Meteorologia na Bahia (INMET) e correspondem a valores médios mensais de precipitação pluviométrica $(\mathrm{mm})$, para a estação 83226, situada no município de Camaçari $\left(12^{\circ} 40^{\prime} \mathrm{S}\right.$ e $\left.38^{\circ} 19^{\prime} \mathrm{W}\right)$. As variáveis hidrológicas: temperatura $\left({ }^{\circ} \mathrm{C}\right)$, salinidade (UPS), pH e saturação de oxigênio (\%), foram coletados utilizando o multi-amostrador Hydrolab Surveyor II. A turbidez (NTU), foi determinada pelo método nefelométrico e a concentração de amônia (mg. $\left.\mathrm{L}^{-1}\right)$, ortofosfato-P (mg. $\mathrm{L}^{-1}$ ) e clorofila a $\left(\mu \mathrm{g} . \mathrm{L}^{-1}\right)$, foram determinadas através das técnicas descritas em Strickland \& Parson (1965). A biomassa do microplâncton foi estimada a partir da amostra total através do método de deslocamento, conforme Kramer et al. (1972).

O ictioplâncton foi coletado através de arrastos oblíquos utilizando-se rede bongo malha 500 $\mu \mathrm{m}$, dotadas de fluxômetro Hydro-Bios, segundo metodologia proposta por Smith \& Richardson (1977). Os arrastos tiveram a duração de 5 minutos e foram realizados a uma velocidade em torno de 1,5 kt. O macrozooplâncton obtido foi fixado em formol a 4 \%, neutralizado com tetraborato de sódio e acondicionado em frascos de $500 \mathrm{~mL}$. O cálculo do volume de água filtrada pela rede foi realizado através da seguinte fórmula: $\mathrm{V}=$ a.n.c, onde: $\mathrm{V}=$ volume de água filtrada $\left(\mathrm{m}^{3}\right)$; $\mathrm{a}=$ área da boca da rede $\left(\mathrm{m}^{2}\right) ; \mathrm{n}=$ número de rotações durante $\mathrm{o}$ arrasto (rot); $\mathrm{c}=$ fator de aferição do fluxômetro $\left(\mathrm{m}_{\text {.rot }}{ }^{-1}\right)$.

A identificação das larvas e jovens de peixes foi realizada ao menor taxon possível, utilizando as seguintes obras: Fahay (1983), Leis \& Remmis (1983), Moser et al. (1984), Okiyama (1988), Olivar; Fortuno (1991) e Ozawa (1995). A sinopse taxonômica geral baseou-se na classificação apresentada em Nelson (1994).

A densidade (organismo.100 $\mathrm{m}^{-3)}$, foi obtida a partir do quociente entre o número total de ovos ou de larvas de peixes obtidos em cada amostra $(\mathrm{N})$ e o volume de água filtrada $(\mathrm{V})$, através da fórmula: $\mathrm{N} / 100 \mathrm{~m}^{3}=(\mathrm{N} / \mathrm{V}) * 100$. A abundância relativa (\%), foi calculada de acordo com a fórmula: $\mathrm{Ar}=$ (Na*100) / NA, onde: Na, é o número total de larvas de peixes de cada família obtido na amostra e NA, é o número total de larvas de peixes na amostra. A frequência de ocorrência (\%), foi calculada pela fórmula: Fo = $($ Ta x 100) / TA, onde Ta, é o número de amostras onde o taxa ocorreu e TA, é o total de amostras. Os critérios adotados para determinação das famílias características foram uma abundância relativa maior que 100/S, sendo S o número de taxa, e uma frequiência de ocorrência maior que $40 \%$.

O tratamento estatístico envolveu a análise de variância que foi empregada visando verificar a existência ou não de diferença significativa entre os 4 cruzeiros para todos as variáveis oceanográficas analisadas. Considerando que a condição para submeter dados amostrais a uma análise de variância paramétrica é a de que suas variâncias não apresentem diferença significativa, aplicou-se o Teste de Bartlett (1937) descrito em Beiguelman (1996), visando testar a homogeneidade das variâncias. Quando as variâncias eram homogêneas, aplicou-se a ANOVA paramétrica de classificação única; para o caso de variâncias heterogêneas, aplicou-se a ANOVA não paramétrica de Kruskal-Wallis (ZAR, 1984). Para comprovar a existência de diferenças significativas entre os cruzeiros foram realizados testes de comparações múltiplas. Para as variâncias homogêneas aplicou-se o teste paramétrico de Tuckey-Kramer; para o caso de variâncias heterogêneas, foi empregado o teste não paramétrico de Dunn (ZAR, op cite). O coeficiente de correlação Pearson foi empregado para verificar a existência de correlações significativas entre a densidade de ovos e de larvas de peixes, e as variáveis oceanográficas. Como este coeficiente varia entre -1 e 1 , coeficientes acima de 0,7 foram considerados significativos. A elaboração de mapas, visando a representação da distribuição espacial de ovos e larvas de peixes foi realizada no programa Surfer for Windows (KEEKLER, 1995). 


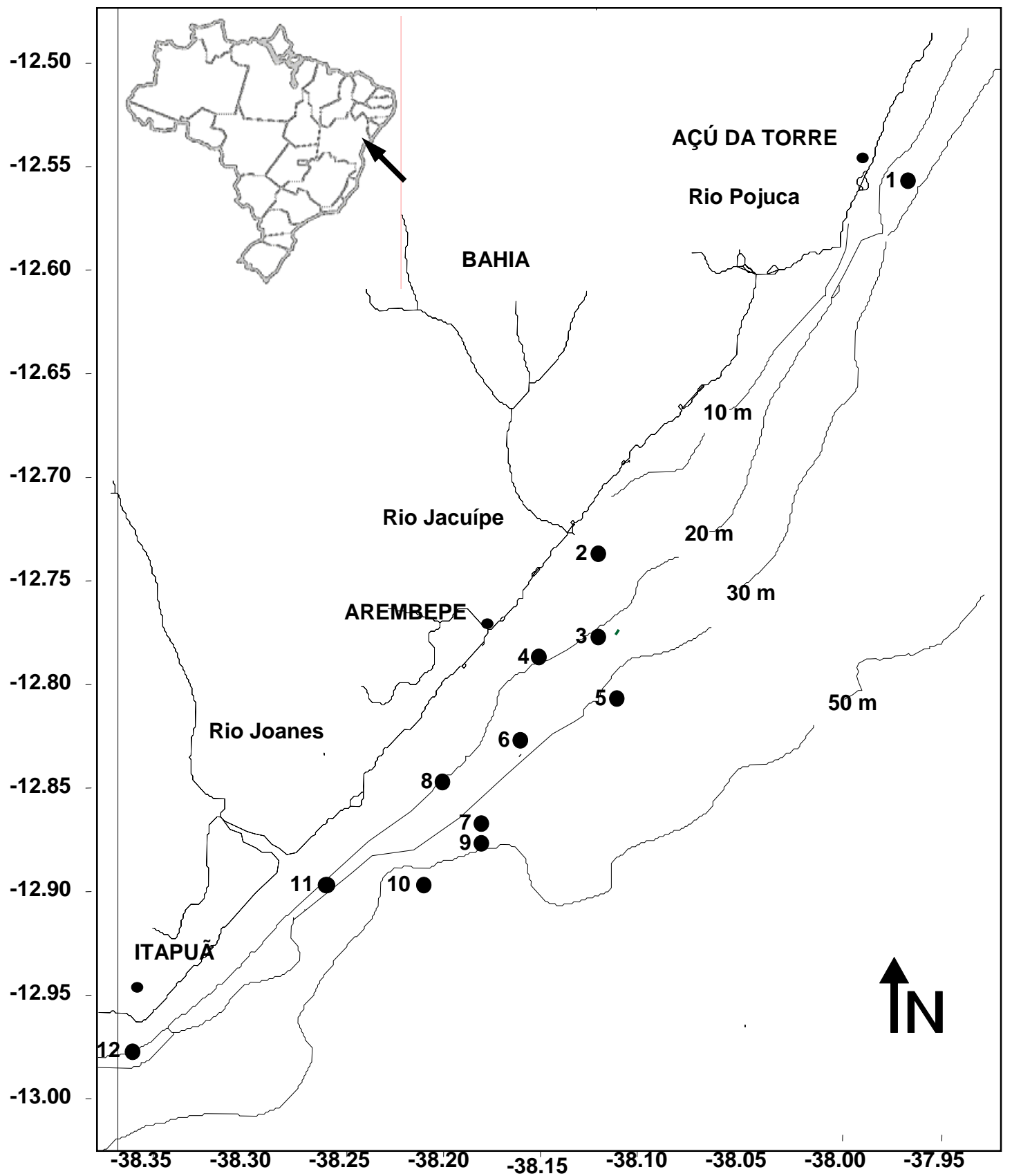

Figura 1 - Mapa de localização das estações de amostragem, no período de maio/1993 a março/1994, entre Açú da Torre e Itapuã, na costa norte da Bahia, Brasil. 


\section{RESULTADOS}

\section{Precipitação pluviométrica}

O padrão de precipitação pluviométrica no litoral norte da Bahia apresentou um período seco e um período chuvoso. O período seco estendeu-se entre setembro/93 e janeiro/94. Nos cruzeiros realizados durante este período, os valores médios de pluviosidade foram de $76,7 \mathrm{~mm}$, em setembro/93 e 18,5 mm, em dezembro/93. Entre maio e agosto/93 e, entre fevereiro e março/94, os índices pluviométricos foram mais elevados, caracterizando o período chuvoso. Durante os cruzeiros deste período os valores de pluviosidade foram de 223,7 mm, em maio/93 e 138,5 mm, em março/94 (Fig.2).

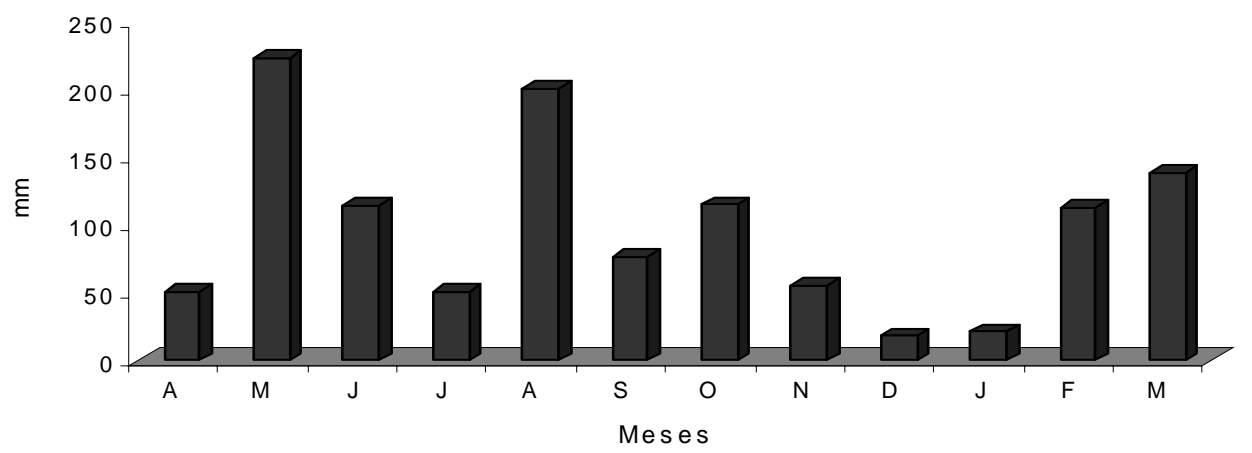

Figura 2 - Variação das médias mensais da precipitação pluviométrica (mm), no período de abril/1993 (A) a março/1994 (M), entre Açú da Torre e Itapuã, Bahia.

\section{Diagrama T-S} T-S (Fig.3).

As massas de água presentes no norte da Bahia podem ser visualizadas através do diagrama

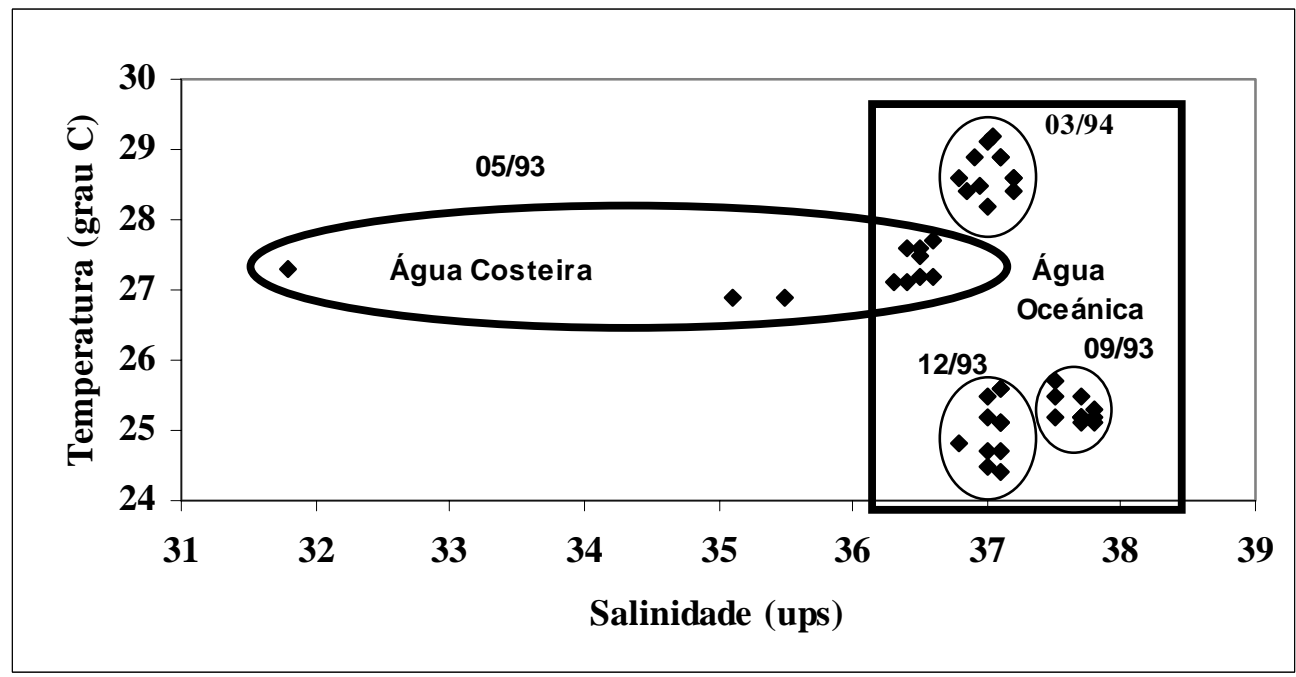

Figura 3 - Caracterização das massas de água, através do Diagrama T/S, no período de maio/1993 a março/1994, entre Açú da Torre e Itapuã, Bahia. 
A massa de água Costeira, com temperatura entre 25,7 e $27,6^{\circ} \mathrm{C}$ e salinidade entre 31,8 e 35,5 , foi formada apenas no mês de maio/93, em função da elevada pluviosidade e conseqüênte aumento da vazão estuarina. Porém, em todos os cruzeiros foi registrada a presença da massa de Água Tropical oceânica, que segundo Garfiled (1990) é caracterizada por temperatura entre 24 e $26^{\circ} \mathrm{C}$ e salinidade entre 36 e 38.

\section{Ocorrência e abundância de larvas}

Foram analisadas 826 larvas e identificadas 12 Ordens, 33 famílias, 35 gêneros e 20 espécies (Apêndice 1). As Tabelas 1 e 2 apresentam os dados de abundância relativa, freqüência de ocorrência e o número de larvas capturadas dos taxa identificados em cada cruzeiro.

No cruzeiro de maio/93 (Tabela 1) foram consideradas características as famílias Engraulidae, Carangidae, Clupeidae e Gerreidae, que representaram, 67,2\% da abundância total. Em setembro/93 as famílias características foram Gerreidae, Haemulidae e Engraulidae, que representaram 44,4\% da densidade total de larvas (Tabela 1). As famílias Gobiidae e Gerreidae foram as únicas características em dezembro/93 (Tabela 2), quando representaram 76,2\% da densidade total. Em março/94 as famílias características foram Carangidae, Clupeidae, Gerreidae e Scombridae, representando $60 \%$ da abundância total de larvas (Tabela 2).

\section{Ocorrência e abundância das famílias características da associação de larvas de peixes}

A abundância relativa das famílias características da associação de larvas de peixes do norte da Bahia, durante o período de maio/93 a março/94 (Fig.4) foram as seguintes: Gobiidae (25\%), Gerreidae (11\%), Engraulidae (11\%), Clupeidae (10\%), Carangidae (7\%), Myctophidae (5\%), Haemulidae (5\%), e Scombridae (3\%).

Os Gobiidae foram representados por 2 gêneros: Gobionellus e Gobiosoma, cuja abundância relativa oscilou entre 66,8\%, em dezembro/93 e 0,6\%, em maio/93. Esta família ocorreu em todos cruzeiros e sua frequência de ocorrência variou entre 83,3\%, em dezembro/93 à apenas 10,0\%, em maio/93 (Tabelas 1 e 2). A família Gerreidae foi representada pela espécie Diapterus rhombeus que também ocorreu em todos os cruzeiros analisados. A sua frequência de ocorrência oscilou entre 75,0\%, em setembro/93, e 42,7\%, em maio/93 (Tabelas 1 e 2). A sua abundância relativa variou entre 20,1\%, em setembro/93 e 4,5\% em maio/93.

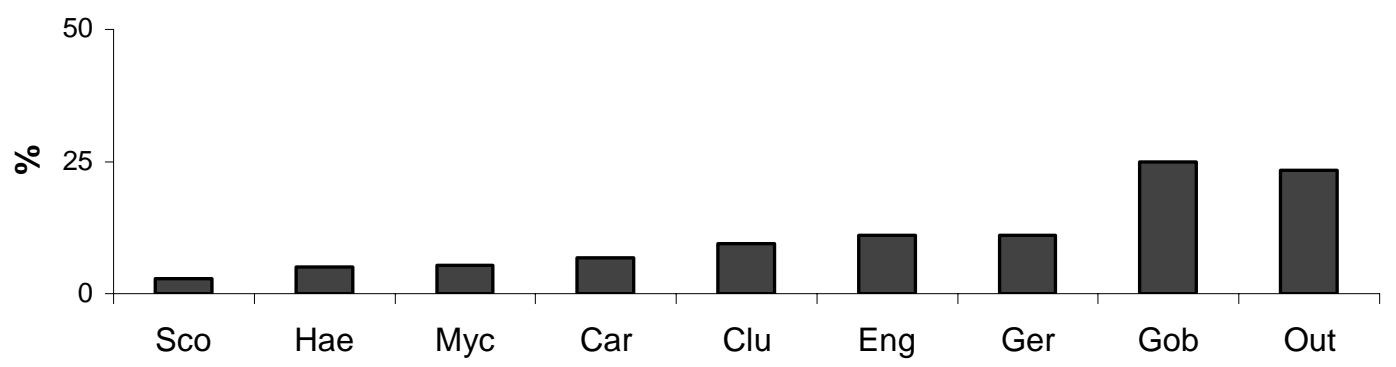

Figura 4 - Abundância relativa das famílias características da associação ictioplanctônica acumulada em 4 cruzeiros, entre Açú da Torre e Itapuã, Bahia. 
Tabela 1 - Dados de captura dos taxa identificados nos cruzeiros realizados em maio de 1993 (Outono) e em setembro de 1993 (Inverno) na região entre entre Açú da Torre e Itapuã, Bahia, onde: número de larvas capturadas $=\mathrm{n}$; abundância relativa $=\%$ e frequência de ocorrência $=$ fo. Valores em negrito indicam famílias características.

\begin{tabular}{|c|c|c|c|c|c|c|c|}
\hline Taxa (maio/1993) & $\mathbf{n}$ & $\%$ & fo & Taxa (setembro/1993) & $\mathbf{n}$ & $\%$ & fo \\
\hline 01. Paralichthidae & 1 & 0,5 & 8,3 & 01. Cynoglossidae & 1 & 0,45 & 8,3 \\
\hline 02. Cynoglossidae & 1 & 0,5 & 8,3 & 02. Soleidae & 2 & 0,9 & 8,3 \\
\hline 03. Gobiidae & 1 & 0,5 & 8,3 & 03. Stromateidae & 1 & 0,45 & 8,3 \\
\hline 04. Serranidae & 1 & 0,5 & 8,3 & 04. Carangidae & 1 & 0,45 & 8,3 \\
\hline 05. Ophidiidae & 1 & 0,5 & 8,3 & 05. Perciformes & 1 & 0,45 & 8,3 \\
\hline 06. Macrouridae & 1 & 0,5 & 8,3 & 06. Scorpaenidae & 1 & 0,45 & 8,3 \\
\hline 07. Tetraodontidae & 3 & 1,6 & 8,3 & 07. Exocoetidae & 4 & 1,8 & 8,3 \\
\hline 08. Hemiramphidae & 2 & 1,0 & 16,7 & 08. Clupeidae & 5 & 2,2 & 8,3 \\
\hline 09. Scombridae & 3 & 1,6 & 16,7 & 09. Bothidae & 10 & 4,5 & 16,7 \\
\hline 10. Haemulidae & 3 & 1,6 & 16,7 & 10. Blennioidei & 2 & 0,9 & 16,7 \\
\hline 11. Sciaenidae & 4 & 2,1 & 16,7 & 11. Sparidae & 2 & 0,9 & 16,7 \\
\hline 12. Sparidae & 10 & 5,3 & 33,3 & 12. Serranidae & 8 & 3,6 & 25 \\
\hline 13. Myctophidae & 18 & 9,6 & 33,3 & 13. Bregmacerotida & 16 & 7,2 & 25 \\
\hline 14. Blenniidae & 5 & 2,7 & 41,7 & 14. Myctophidae & 11 & 4,9 & 25 \\
\hline 15. Gerreidae & 8 & 4,3 & 41,7 & 15. Gonostomatidae & 6 & 2,7 & 33,3 \\
\hline 16. Clupeidae & 23 & 12,2 & 41,7 & 16. Blenniidae & 10 & 4,5 & 33,3 \\
\hline 17. Carangidae & 39 & 20,8 & 50 & 17. Gobiidae & 43 & 19,3 & 33,3 \\
\hline \multirow[t]{3}{*}{ 18. Engraulidae } & 64 & 34,2 & 66,7 & 18. Haemulidae & 21 & 9,4 & 50 \\
\hline & & & & 19. Engraulidae & 33 & 14,8 & 50 \\
\hline & & & & 20. Gerreidae & 45 & 20,2 & 75 \\
\hline Total & 188 & 100 & & & 222 & 100 & \\
\hline
\end{tabular}

A família Engraulidae, representada pela espécie Lycengraulis grossidens, apresentou valores máximos de abundância relativa (34,2\%) e frequência de ocorrência (66,7\%), em maio/93 (Tabelas 1 e 2). Os Clupeidae, representados pelas espécies Harengula clupeola e Opisthonema oglinum, apresentaram abundância relativa oscilando entre 33,4\% em março/94 e 0\% em dezembro/93. A sua frequência de ocorrência variou entre 58,3\%, em março/94 e 0\%, em dezembro/93 (Tabelas 1 e 2). 
Tabela 2 - Dados de captura dos taxa identificados nos cruzeiros realizados em dezembro de 1993 e em março de 1994 (verão), na região entre entre Açú da Torre e Itapuã, Bahia, onde: número de larvas capturadas $=n$; abundância relativa $=\%$ e frequência de ocorrência $=$ fo. Valores em negrito indicam famílias características.

\begin{tabular}{|c|c|c|c|c|c|c|c|}
\hline Taxa (dezembro/1993) & $\mathbf{n}$ & $\%$ & fo & $\begin{array}{c}\text { Taxa } \\
\text { (março/1994) }\end{array}$ & $\mathbf{n}$ & $\%$ & fo \\
\hline 01. Stromateidae & 1 & 0,4 & 8,3 & 01. Tetraodontidae & 1 & 0,5 & 8,3 \\
\hline 02. Scombridae & 1 & 0,4 & 8,3 & 02. Bothidae & 1 & 0,5 & 8,3 \\
\hline 03. Blennioidei & 1 & 0,4 & 8,3 & 03. Blenniidae & 1 & 0,5 & 8,3 \\
\hline 04. Exocoetidae & 1 & 0,4 & 8,3 & 04. Scaridae & 1 & 0,5 & 8,3 \\
\hline 05. Triglidae & 1 & 0,4 & 8,3 & 05. Sciaenidae & 1 & 0,5 & 8,3 \\
\hline 06. Gonostomatidae & 1 & 0,4 & 8,3 & 06. Scorpaenidae & 1 & 0,5 & 8,3 \\
\hline 07. Balistidae & 2 & 0,9 & 8,3 & 07. Ophidiidae & 1 & 0,5 & 8,3 \\
\hline 08. Pomacanthidae & 2 & 0,9 & 8,3 & 08. Synodontidae & 1 & 0,5 & 8,3 \\
\hline 09. Blenniidae & 3 & 1,3 & 8,3 & 09. Gonostomatidae & 1 & 0,5 & 8,3 \\
\hline 10. Sciaenidae & 2 & 0,9 & 16,7 & 10. Ophichthidae & 1 & 0,5 & 8,3 \\
\hline 11. Sparidae & 4 & 1,7 & 16,7 & 11. Serranidae & 3 & 1,7 & 8,3 \\
\hline 12. Serranidae & 10 & 4,4 & 16,7 & 12. Lutjanidae & 8 & 4,3 & 8,3 \\
\hline 13. Tetraodontidae & 7 & 3,0 & 33,3 & 13. Monacanthidae & 2 & 1,1 & 16,7 \\
\hline 14. Myctophidae & 10 & 4,4 & 33,3 & 14. Exocoetidae & 2 & 1,1 & 16,7 \\
\hline 15. Haemulidae & 9 & 3,9 & 50 & 15. Stromateidae & 5 & 2 & 16,7 \\
\hline 16. Gerreidae & 21 & 9,2 & 50 & 16. Myctophidae & 3 & 1,7 & 16,7 \\
\hline \multirow[t]{10}{*}{ 17. Gobiidae } & 154 & 67 & 83,3 & 17. Blennioidei & 16 & 8,7 & 16,7 \\
\hline & & & & 18. Haemulidae & 3 & 1,7 & 25 \\
\hline & & & & 19. Engraulidae & 3 & 1,7 & 25 \\
\hline & & & & 20. Gobiidae & 4 & 2,2 & 25 \\
\hline & & & & 21. Não Identif. & 16 & 7,8 & 25 \\
\hline & & & & 22. Percoidei & 2 & 1,1 & 33,3 \\
\hline & & & & 23. Gerreidae & 10 & 5,6 & 50 \\
\hline & & & & 24. Scombridae & 16 & 9 & 50 \\
\hline & & & & 25. Clupeidae & 59 & 33,4 & 58,3 \\
\hline & & & & 26. Carangidae & 24 & 12 & 66,7 \\
\hline$\overline{\text { Total }}$ & 230 & 100 & & & 186 & 100 & \\
\hline
\end{tabular}

A família Carangidae (Tabelas 1 e 2) esteve representada por 3 espécies: Caranx bartholomei, Caranx chrysus, e Chloroscombrus chrysurus, que apresentaram abundância relativa máxima de 20,8\%, em maio/93 e a sua maior frequência de ocorrência, em março/94 (66,7\%). Os Myctophidae foram representados por 4 gêneros: Benthosema, Ceratoscopelus, Diaphus e Lampadena, que apresentaram abundância relativa entre 9,6\% (maio/93) e 1,7\% (março/94). A frequência de ocorrência desta família atingiu 33,3\%, em dezembro/93, 25\%, em maio/93 e setembro/93 e 16,7\% em março/94 (Tabelas 1 e 2).

A família Haemulidae, representada pelo gênero Haemulon, registrou uma abundância relativa entre $9,7 \%$, em setembro/93 e 1,6\%, em março/94. A sua frequência de ocorrência variou entre $50 \%$ em setembro e 16,7\% em maio/93 (Tabelas 1 e 2). A família Scombridae, representada pelas espécies Scomberomorus cavalla e Auxis thazard, apresentou frequência de ocorrência variando entre 50\%, em março/94, e $0 \%$ em setembro/93 (Tabelas 1 e 2). Os Scombridae apresentaram uma abundância relativa entre 9,0\%, em março/94 e 0\%, em setembro/93. 


\section{Distibuição espacial e sazonal}

A densidade média de ovos de peixes encontrada entre maio/93 e março/94, foi de 189 ovos.100 $\mathrm{m}^{-3}$. As maiores densidade foram encontradas na porção sul da área de estudo. A maior amplitude na distribuição espacial da densidade de ovos de peixes ocorreu no cruzeiro de setembro/93, com 26 ovos/100 $\mathrm{m}^{3}$, na estação 2 e 537 ovos/100 ms, na estação 12 . No cruzeiro de março/94, a menor densidade foi encontrada na estação 7 (57 ovos/100 $\left.\mathrm{m}^{3}\right)$ e a maior densidade na estação $12\left(603\right.$ ovos $\left./ 100 \mathrm{~m}^{3}\right)$. Em maio/93 a amplitude variou entre 24 ovos/100 $\mathrm{m}^{3}$ (estação 4) e 381 ovos/100 m (estação 9). Em dezembro/93 foi encontrada a menor amplitude na densidade de ovos de peixes; o pico na densidade ocorreu na estação 7, que registrou 363 ovos/100 m³ (Fig.5). O pico sazonal na densidade média de ovos de peixes foi encontrado no mês de março/94 (226 ovos/100 $\mathrm{m}^{3}$ ) (Fig.6). A análise estatística não verificou diferença significativa entre os diferentes cruzeiros (ANOVA, p > 0,0,5). A densidade média de ovos de peixes apresentou coeficientes de correlação Pearson significativos com: biomassa de microplâncton $(0,98)$, temperatura $(0,86)$, ortofosfato $(0,8)$, biomassa fitoplanctônica $(0,71)$ e turbidez $(0,61)$.

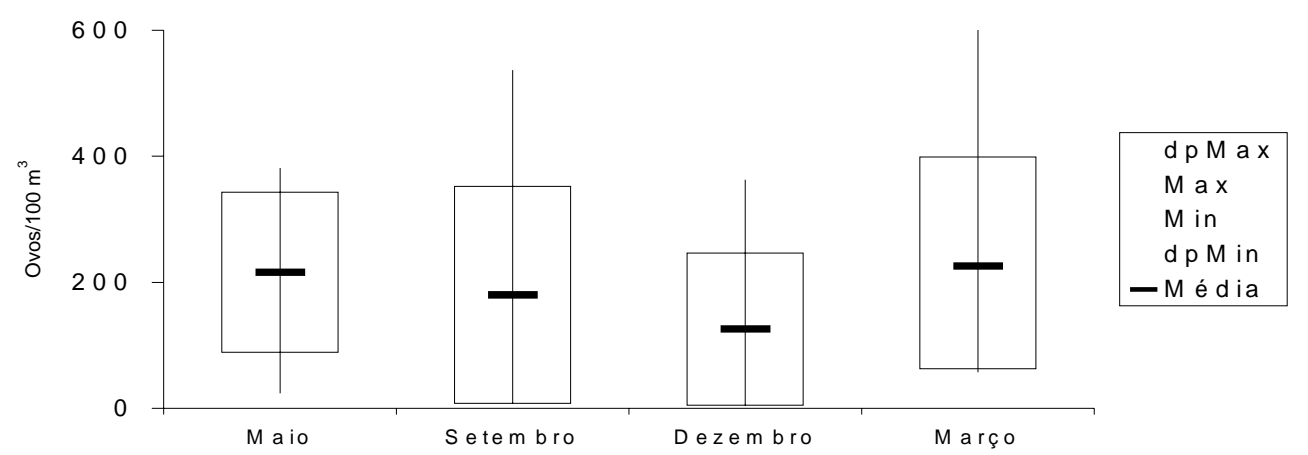

Figura 5 - Variação sazonal dos valores médios da densidade de ovos de peixes/100 $\mathrm{m}^{3}$, no período de maio/1993 a março/1994, entre Açú da Torre e Itapuã, Bahia.

As maiores amplitudes na distribuição espacial da densidade total de larvas de peixes/100 $\mathrm{m}^{3}$, foram encontradas nos cruzeiros de setembro/93 (0 a 300 larvas/100 $\left.\mathrm{m}^{3}\right)$ e dezembro/93 $(0$ a 271 larvas/100 $\left.\mathrm{m}^{3}\right)$. Os cruzeiros de maio/93 (0 a 93 larvas/100 $\left.\mathrm{m}^{3}\right)$ e março/94 (6 a 80 larvas/100 $\mathrm{m}^{3}$ ) apresentaram as menores amplitudes (Fig. 7). Em setembro/93, o pico na densidade de larvas foi registrado na estação 10, que registrou 300 larvas/100 $\mathrm{m}^{3}$. No cruzeiro de dezembro/93 a maior densidade foi encontrada na estação 7. Em maio/93, o pico se deu na estação 9 e, em março/94, na estação 5. Entre maio/93 e março/94 foi encontrada uma densidade média de 41 larvas de peixes $/ 100 \mathrm{~m}^{3}$. As larvas de peixes apresentaram maiores densidades nas estações localizadas na porção sul da área amostral. O pico sazonal na densidade média de larvas de peixes foi obtido no mês de setembro/93 (57 larvas/100 $\mathrm{m}^{3}$ ). No mês de dezembro/93 foi registrada uma densidade média de 42 larvas $/ \mathrm{m}^{3}$. Os menores valores (Fig.8) foram registrados em maio/93 (28 larvas/100m³) e março/94 (35 larvas/100m ${ }^{3)}$.

A análise estatística não encontrou diferença significativa entre os cruzeiros estudados (ANOVA, p > 0,05). A densidade média de larvas de peixes apresentou coeficientes de correlação Pearson significativos com saturação de oxigênio $(0,99)$, turbidez $(-0,91)$, salinidade $(0,89)$, ortofosfato $(-0,86)$, biomassa fitoplanctônica $(-074)$, amônia $(-0,71)$ e temperatura $(-0,66)$. 

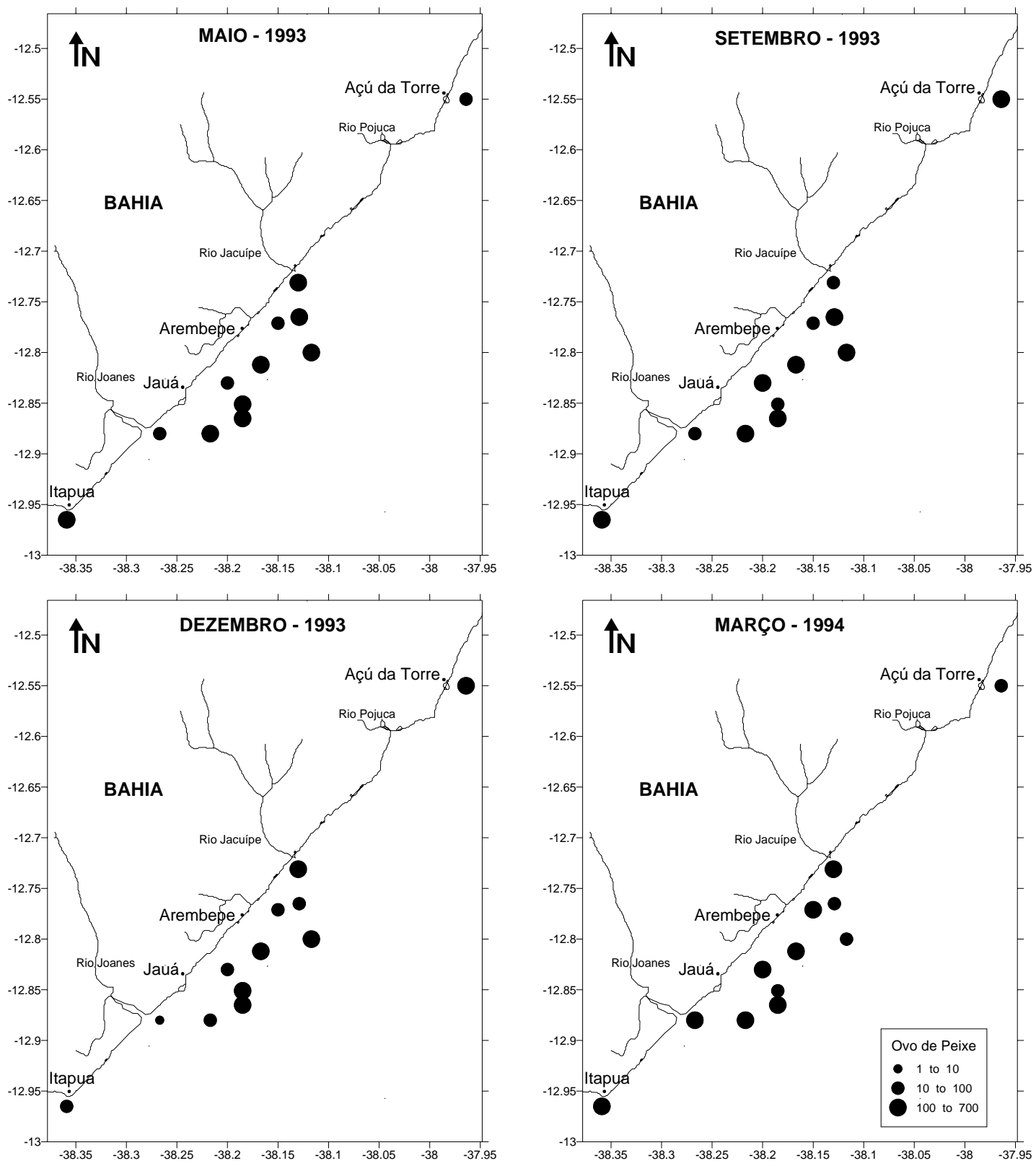

Figura 6 - Distribuição espacial e sazonal da densidade de ovos de peixes $/ 100 \mathrm{~m}^{3}$, no período de maio/1993 a março/1994, entre Açú da Torre e Itapuã, Bahia.

\section{DISCUSSÃo}

O regime pluviométrico do norte da Bahia apresentou dois períodos distintos: um período chuvoso, característico do outono (maio/1993) e verão (março/1994) e um período seco, dominante no inverno (setembro/1993) e primavera (dezembro1993). O período seco foi caracterizado por elevada salinidade, saturação de oxigênio e pH, baixa turbidez e baixas concentrações de nutrientes 
(amônia e ortofosfato), biomassa fitoplanctônica e biomassa zooplanctônica e pelo predomínio da massa de água Tropical Oceânica. O período chuvoso foi caracterizada por menores salinidades e saturação de oxigênio, altas temperaturas e valores elevados de turbidez, concentração de amônia, ortofosfato e biomassa de fitoplâncton e zooplâncton, onde, além da presença de massa de água Oceânica, também foi verificada a ocorrência de massa de água Costeira (MAFALDA JR., 2000).

A costa norte da Bahia apresentou riqueza taxonômica moderada com larvas de 33 famílias de peixes que representam um acréscimo de 17 famílias, em relação ao norte da Baía de Todos os Santos (MAFALDA JR., 1995). A costa Central do Brasil, entre Belmonte e Cabo Frio, onde foram identificadas 77 famílias (NONAKA et al., 2000) e a plataforma continental entre Recife e Fortaleza, com 74 famílias identificadas (EKAU et al., 1999), constituem as áreas da costa Brasileira com maior riqueza ictioplanctônica. Sinque \& Muelbert (1997), apontam a ocorrência de 57 famílias no ictioplâncton nerítico e oceânico do Rio Grande do Sul.

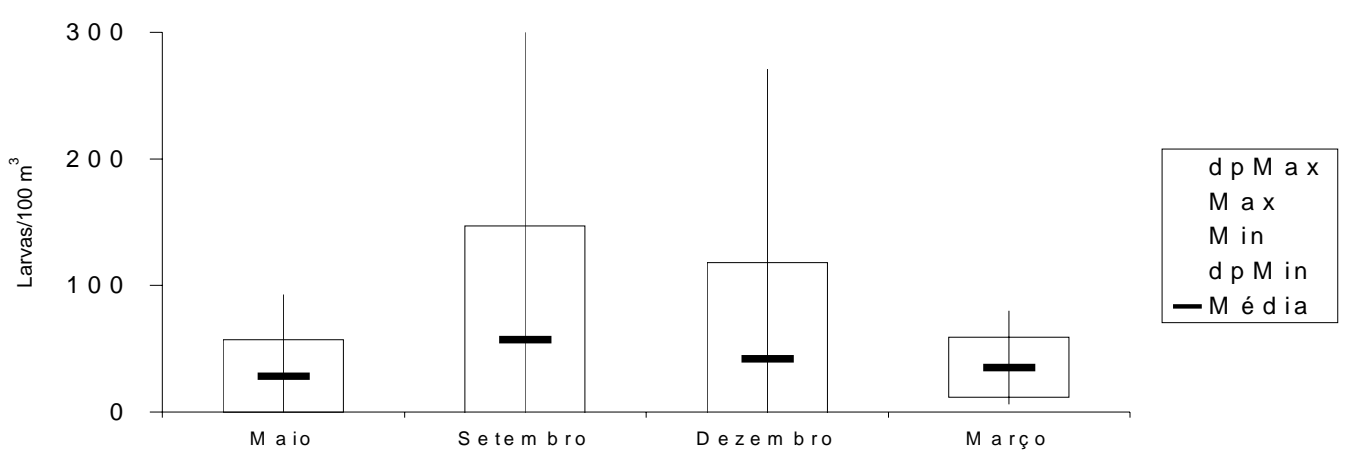

Figura 7 - Variação sazonal dos valores médios da densidade de larvas de peixes $/ 100 \mathrm{~m}^{3}$, no período de maio/1993 a março/1994, entre Açú da Torre e Itapuã, Bahia.

No litoral norte da Bahia entre as 33 famílias registradas, 13 estão associadas a recifes de corais (39,4\%), 9 são demersais (27,3\%), 8 são epipelágicas (24,2\%) e apenas 3 famílias são mesopelágicas $(9,1 \%)$. Contudo, entre as 8 famílias características do ictioplâncton, 5 são epipelágicas, 2 estão associadas a recifes e 1 é mesopelágica, indicando a dominância numérica dos pelágicos. Ou seja, apesar de maior riqueza das famílias associadas a recifes de corais e das famílias de demersais, a sua abundância relativa é inferior a das famílias pelágicas, sugerindo diferentes estratégias reprodutivas. Segundo Nonaka (2000), as diferenças na distribuição das larvas associadas a recifes podem ser atribuídas à local da desova, cuidado parental e comportamento das larvas nas primeiras fases do ciclo de vida.

Assim, entre as famílias que caracterizam o ictioplâncton da costa norte da Bahia se encontram as famílias epipelágicas (Engraulidae, Clupeidae, Gerreidae, Carangidae e Scombridae), as famílias associadas a recifes de corais (Haemulidae e Gobiidae), e os mesopelágicos (Myctophidae). O ictioplâncton da Baía de Todos os Santos (MAFALDA JR., 1995), onde predomina a massa de água Costeira (MAFALDA JR. et al., 2002a), caracteriza-se pela ausência de mesopelágicos. Porém, em função da predominância de epipelágicos e recifais, a semelhança na composição da ictiofauna larval com o norte da Bahia, indica a ocorrência de uma comunidade costeira comum aos dois ambientes. Nas águas oceânicas, em frente a costa norte da Bahia, a predominância é de mesopelágicos como Myctophidae e Gonostomatidae, embora também sejam registradas as famílias epipelágicas e as associadas a recifes (SILVA, 1997; PINTO et al., 2002). 

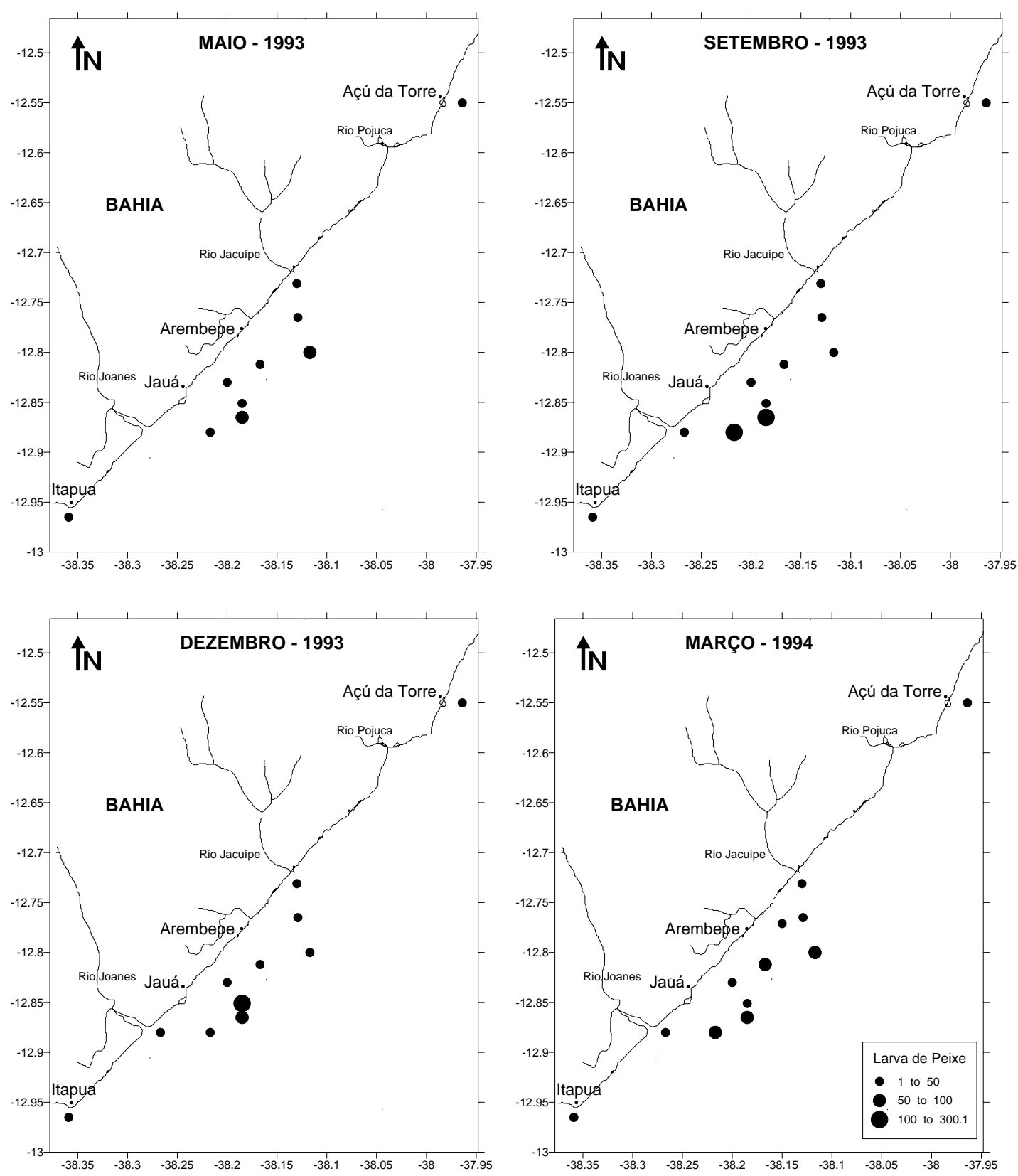

Figura 8 - Distribuição espacial e sazonal da densidade de larvas de peixes/100 $\mathrm{m}^{3}$, no período de maio/1993 a março/1994, entre Açú da Torre e Itapuã, Bahia.

Na área de estudo, assim como em outras regiões costeiras do nordeste Brasileiro (EKAU; WESTHAUS-EKAU, 1996; EKAU; MATSUURA, 1996; NONAKA, 2000), também vem sendo registrado uma maior riqueza de famílias associadas a recifes de corais, em relação a riqueza de famílias pelágicas. Porém, nas águas oceânicas da Zona Econômica Exclusiva, predominam os 
mesopelágicos, como Myctophidae Gonosotomatidae e Sternoptychidae (CARROZO et al., 1999; Lessa et al., 1999; Mafalda Jr. et al., 2002b; Nonaka et al., 2000), indicando forte influência da Água Central do Atlântico Sul (ACAS), que ocorre abaixo da termoclina, em torno dos $150 \mathrm{~m}$ de profundidade (EKAU; MATSUURA, 1996). Na zona costeira do nordeste Brasileiro os padrões observados são consequência da existência de um ambiente pelágico oligotrófico, onde as áreas mais produtivas estão associadas a exportação do material de manguezais, via descarga fluvial, e a ressurgência de borda de plataforma (MEDEIROS et al, 1999), onde são encontradas elevadas densidades de ovos e larvas de peixes (EKAU; WESTHAUS-EKAU, 1996; EKAU; MATSUURA, 1996; NONAKA, 2000).

As densidade de ovos de peixes, encontradas no litoral norte da Bahia, foram bastante superiores aquelas verificadas na Zona Econômica Exclusiva Nordestina por Silva (1997) e Carrozo et al. (1999), indicando a sua utilização como sítio de desova. Na Baía de Todos os Santos (MAFALDA JR., 1995) e na costa o norte do Pernambuco, também foram registradas densidades elevadas de ovos de peixes (EKAU; WESTHAUS-EKAU, 1996).

Na costa norte da Bahia, a existência de taxa pelágicos, demersais e associados a recifes, que habitam a plataforma continental, além de espécies mesopelágicas que apesar de viverem próximas ao talude, se aproximam da costa para a reprodução, tornam a plataforma continental um ambiente relativamente abundante em ictioplâncton. Elsayed \& Hamid (1977) apontam a importância das águas costeiras do Egito para a reprodução de peixes pelágicos (Engraulidae, Carangidae e Pomatomidae) e recifais (Serranidae e Sparidae). No estreito de Gibraltar e no mar de Alborán, as elevadas densidades de ovos de peixes são atribuídas a reprodução de espécies pelágicas e demersais típicas da plataforma continental (RUBIN, 1997a), já que as espécies mesopelágicas realizam desova oceânica (RUBIN, 1997b).

A correlação positiva da densidade de ovos de peixes com a temperatura da água, concentração de nutrientes, biomassa fitoplanctônica e biomassa zooplanctônica é consequência da localização do sítio de desova no litoral norte da Bahia, principalmente durante o período chuvoso, em relação ao período seco. Ou seja, as características oceanográficas envolvendo a temperatura elevada e a disponibilidade de alimento tornam os períodos chuvosos mais favoráveis para a formação do sítio de desova do que o período seco, quando foram observadas águas relativamente mais frias e com menor concentração de organismos planctônicos. A associação entre ovos de peixes e temperatura de superfície pode ser atribuída ao fato da atividade de desova estar relacionada com temperaturas mais elevadas (BORKIN, 1992).

A elevada amplitude encontrada na densidade de ovos e de larvas de peixes é consequência da variabilidade espacial e sazonal. As densidade de larvas de peixes, encontradas no norte da Bahia, também foram superiores aquelas verificadas na Zona Econômica Exclusiva oceânica adjacente por Silva (1997) e na Baía de Todos os Santos por Mafalda Jr. (1995). Níveis semelhantes de densidade larval foram obtidos na regiao costeira entre Rio Formoso (AL) e João Pessoa (PB), por Ekau \& Westhaus-Ekau (1996).

A densidade de larvas de peixes que foram encontradas no litoral norte da Bahia, em relação as áreas oceânicas do nordeste brasileiro, indica a sua utilização como sítio de crescimento, da mesma forma que em outras regiões costeiras, principalmente em frente aos estuários. A correlação da densidade de larvas de peixes, com as variáveis oceanográficas, destaca a importância da saturação de oxigênio e da salinidade para a formação dos sítios de criação, principalmente durante o período seco. Além disto, a correlação negativa observada com a biomassa de fitoplâncton provavelmente está indicando atividade alimentar das larvas de peixes sobre estes organismos.

Como as larvas e pós larvas são limitadas em seus movimentos, o ictioplâncton está sujeito às massas de água e correntes marinhas, evidenciando a importância das caracterísiticas hidrológicas sobre sua distribuição (CIECHONSKI, 1981). LAPRISE; PEPIN (1995) consideram a temperatura e secundariamente a salinidade como principais fatores que controlam a distribuição espacial e temporal de ovos e larvas de peixes nas águas costeiras de Newfoundland e Labrador no Canadá. No norte da Bahia, a temperatura foi mais importante na distribuição dos ovos de peixes, 
em função das águas mais quentes encontradas durante o período chuvoso, enquanto que, a salinidade foi mais importante na distribuição das larvas de peixes, devido as águas mais salinas verificadas no período seco. Ou seja, a utilização do litoral norte da Bahia, como sítio de desova e crescimento de larvas de peixes, foi verificada em todas as épocas do ano, com maior densidade de ovos no período chuvoso e maior densidade de larvas durante o período seco.

\section{AgRAdecimentos}

Agradecemos ao PICD-CAPES, pela bolsa de estudos que viabilizou a realização deste trabalho, como parte consitutinte da Tese de Doutorado em Oceanografia Biológica, desenvolvida no Departamento de Oceanografia da FURG. Ao CEPEMAR (Centro de Pesquisas do Mar) pelo apoio logísitico e ao Instituto de Biologia da Universidade Federal da Bahia, pelo afastamento concedido para realizaçao do Doutorado. A Sra. Lúcia Marzinha pelo auxílio na identificaçao das larvas de peixes.

\section{REFERÊNCIAS BIBLIOGRÁFICAS}

Beiguelman, B. Curso Prático de Bioestatística. Ribeirão Preto: Revista Brasileira de Genética, 1996. 182 p.

BORKIN, I. V. Ichthyoplankton of the coastal waters of western Spitsbergen. J. Ichthyol., v. 32, 145-153, 1992.

BRANDINI, F. P.; LOPES, R. M.; GUTSEIT, K. S.; SPACH, H. L.; SASSI, R. Planctonologia na Plataforma Continental do Brasil. Diagnose e Revisão Bibliográfica. Rio de Janeiro: REVIZEE. MMA, CIRM, FEMAR, 1997. 196 p.

CARROZZO, G.; MAFALDA JR. P.; PINTO, N. C. T.; LIMA, D. L.; VELAME, M. P. Distribuição espacial e temporal do ictioplâncton ao longo da Zona Econômica Exclusiva Nordestina, entre Salvador, Bahia e Rio Parnaíba, Piauí. In: XVIII CONGRESSO

LATINOAMERICANO SOBRE CIÊNCIAS DEL MAR, 18. 1999, Trujillo, Anais... Trujillo 1999, p. 399-400.

CASTILLO, G.; MUNOZ, H.; GONZALEZ, H.; BERNAL, P. Daily analysis of abundance and size variability of fish larvae in relation to oceanic water intrusions in coastal areas. Biologia Pesquera, v. 20, p. 21-35, 1991.

CIECHOMSKI, J. D. Ictioplâncton. In: BOLTOVOSKOY, D. (Ed.). Atlas del Zooplancton del Atlantico Sudoccidental y Metodos de Trabajo con el Zooplancton Marino. Mar del Plata: INIDEP, 1981. p. 829-860.

DOYLE, M. J.; MORSE, W. W.; KENDALL,Jr., A. W. A comparision of larval fish assemblages in the temperate zone of northeast Pacific and the northwest Atlantic ocean. Bull. Mar. Sci., v. 53. p. 588-644, 1993.

EKAU, W.; MATSUURA, Y. Diversity and distribution of ichthyoplankton in the continental shelf waters of East Brazil. In: EKAU, W; KNOPPERS, B. (Ed.) Sedimentation Processes and Productivity in the Continental Shelf Waters off East and Northeast Brazil. Joint Oceanographic Projects, Cruise Report and First Results. Bremen: Center of Tropical Marine 
Ecology, 1996. p. 135-147.

EKAU, W.; WESTHAUS-EKAU, P. Ichthyoplankton distribution and community structure. Influence of mangroves on diversity and production of the costal waters off Northeast Brazil. In: EKAU, W.; KNOPPERS, B. (Ed.) Sedimentation Processes and Productivity in the Continental Shelf Waters off East and Northeast Brazil. Joint Oceanographic Projects, Cruise Report and First Results. Bremen: Center of Tropical Marine Ecology, 1996. p. 91-95.

EKAU, W.; WESTHAUS-EKAU, P.; MEDEIROS, C. Large scale distribution of fish larvae in the continental shelf waters off North-East Brazil. Arch. Fish. Mar. Res., v. 47, n. 2/3, p. 183-200, 1999.

ELSAYED EL-HEHYAWI, M. L.; HAMID, E. M. The distribution, abundance and some ecological aspects of the ichthyoplankton in the S.E. Mediterranean waters. Bull. Inst. Ocean. \& Fish., A.R.E., v. 7, 91-115, 1977.

FAHAY, M. P. Guide to the early stages of marine fishes occurring in the western North Atlantic Ocean, Cape Hattaras to the southern Scotian Shelf. J. Northwest Atl. Fish. Sci., v. 4, p. 1-423, 1983.

FRANK, K. T.; LEGGETT, W. C. Multispecies larval fish associations: accident or adaptation. Can. J. Fish. Aquat. Sci., v. 40, p. 754-762, 1983.

GARFIELD III, N. The Brazil current at subtropical latitudes. Rhode Island, 1990, 122f. Thesis (Doctor of Philosophy). University of Rhode Island.

KEEKLER, D. SURFER for Windows. Version 6. User's Guide. 1995.

KRAMER, D.; KALIN, M. J.; STEVENS, E. G.; THAIKILL, J. R.; ZWEIFEEL, J. R. Collecting and processing data on fish eggs and larvae in the California Current region. U.S. Dept. of Commerce, NOAA Tech. Rept, NMFS Circ., v. 370, p. 1-38, 1972.

LEIS, J. M. Larval fish assemblages near Indo-Pacific coral reefs. Bull. Mar. Sci., v. 53, p.362-392, 1993.

LEIS, J. M.; REMMIS, D. S. The Larvae of Indo-Pacific Coral Reef Fishes. Sydney: New South Wales University Press, 1983. 269 p.

LESSA, R. P.; MAFALDA JR., P.; ADVÍNCULA, R.; LUCCHESI, R. B.; BEZERRA JR., J. L.; VASKE JR., T.; HELLEBRANDT, D. Distribution and abundance of ichthyoneuston at seamounts and islands off north-eastern Brazil. Arch. Fish. Mar. Res., v. 47, n. 2/3, p. 133-146, 1999.

MAFALDA JR., P. Distribuição e abundância do ictioplâncton da costa norte da Bahia e suas relações com as condições oceanográficas. Rio Grande, 2000, 135f. Tese (Doutorado) Departamento de Oceanografia. Fundação Universidade Federal do Rio Grande.

MAFALDA JR. P.; SOUZA, P. M. M.; SILVA, E. M. Estrutura hidroquímica e biomassa planctônica no norte da baía de Todos os Santos, Bahia, Brasil. Tropical Oceanography, Recife, v. 30, n. 2, p. 117-137, 2002a.

MAFALDA JR. P.; VELAME, M. P. B.; SOUZA, C. S.; PINTO, N. C. T.; SOUZA, P. M. M. 
Distribuição em larga escala de ovos e larvas de peixes na Zona Econômica Exclusiva do Nordeste do Brasil. Informativo CIRM, v. 14, n. 1, p. 8, 2002b.

MAFALDA JR., P. Pesquisa de Índices Ecotoxicológicos nas Associações Planctônicas. In:

PESO-AGUIAR, M. C. (Ed.) Programa de Monitoramento dos Ecossistemas ao norte da Baía de Todos os Santos, Salvador, Universidade Federal da Bahia, Relatório Técnico Final, 1995. p. 1-60.

MAFALDA JR, P.; SILVA, V. R. F. Caracterização do ictioplâncton do sistema estuarino-lagunar de Jequiá, Alagoas. Bol. Estud. Ciênc. Mar, v. 9, p. 89-104, 1996.

MAFALDA JR., P.; LESSA, R. P.; KLOPFER, M.; VELAME, M. P. Abundância de larvas de Pleuronectiformes nos bancos oceânicos da Cadeia Norte Brasileira, durante o verão de 1995. In: SEMANA NACIONAL DE OCEANOGRAFIA, 10. 1999, Itajaí, Anais... Itajaí 1997, p.119-121.

MAFALDA JR., P.; SINQUE,C.; CARROZZO, G. Distribuição e abundância do ictioplâncton entre Açú da Torre e Itapuã, Bahia, Brasil. XVIII CONGRESSO LATINOAMERICANO SOBRE CIÊNCIAS DEL MAR, 18. 1999, Trujillo, Anais... Trujillo 1999, p. 390-392.

MEDEIROS, C.; MACÊDO, S.; FEITOSA, F.; KOENING, M. L. Hydrography and phytoplankton biomass and abundance of North-east Brazilian waters. Arch. Fish. Mar. Res., v. 47, n. 2-3, p. 133$151,1999$.

MOSER, H. G.; RICHARDS, W. J.; COHEN, D. M.; FAHAY, M. P.; KENDALL JR., A. W.; RICHARDSON, S. L. Ontogeny and systematics of fishes. Am. Soc. Ichthy. Herpert. Sp. Pub. v. 1, p. 1-760, 1984.

NELSON, J. S. Fishes of the World. New York: John Wiley \& Sons, 1994. 599 p.

NONAKA, R. H. Distribuição e abundância de larvas de peixes marinhos da região do Banco de Abrolhos e águas adjacentes, e suas relações com as condições oceanográficas. São Paulo, 2000, 143f. Dissertação (Mestrado), Universidade de Sao Paulo.

NONAKA, R. H.; MATSUURA, Y.; SUZUKI, K. Seasonal variation in larval fish assemblages in the Abrolhos Bank region off eastern Brazil. Fish. Bull., v. 98, p. 767-784, 2000.

OKIYAMA, M. An Atlas of the Early Stage Fishes in Japan. Tokyo: Tokai University Press, 1988. $1154 \mathrm{p}$.

OLIVAR, M. P.; FORTUÑO, J. M. Guide to ichthyoplankton of the Southeast Atlantic (Benguela Current Region). Sci.Mar., v. 55, n. 1, p. 1-383, 1991.

OZAWA, T. Studies on the Oceanic Ichthyoplankton in the Western North Pacific. Hakozaki: Kyushu University Press, 1995.

PAREDES, J. F. Evaluation of the environmental effects of the industrial effluents from Tibrás Titânio do Brasil in the area under influence of its underwater outfalls. In: INTERNATIONAL SPECIALIZED CONFERENCE ON MARINE DISPOSAL SYSTEM, 1991, Lisboa, Anais... Lisboa 1991, p.105-125.

PAREDES, J. F.; FIGUEIREDO, G.; ROCHA, W. L. C. Hydrobiological studies of the drainage system of the Bananeiras reservoir, the Rivers Paraguaçú and Jacuípe. In: TRANSPORT OF 
CARBON AND NUTRIENTS IN LAKES AND ESTUARIES, 1993, Hamburg, Anais... Hamburg 1993, p.23-39.

PAREDES, J. F.; FREITAS, R. R. Dosagem de ferro nos sedimentos e interpretação da formação de espumas no estuário do Rio Jacuípe quando da ocasião de cheias. Ciência e Cultura, v. 32, n. 10, p. 84-87, 1980.

PINTO, N. C. T.; MAFALDA JR. P.; MEDEIROS, C.; MOURA, G.; SOUZA, C. S. Distribuição de larvas de Katsuwonus pelamis (Pisces, Scombridae), em larga escala, na Zona Econômica Exclusiva do Nordeste do Brasil. Tropical Oceanography, Recife, v. 30, n. 2, p. 171-184, 2002.

RUBIN, J. P. Ictiopláncton estival en el mar de Alborán y estrecho de Gibraltar: composición cualitativa-cuantitativa y distribution espacial (julio de 1993). Publ. Espec. Inst. Esp. Oceanogr., v. 24, p. 27-41, 1997a.

RUBIN, J. P. Las larvas de peces mesopelágicos del mar de Alborán. Resultados de la campana Ictio. Alborán 0793 y revisión historica. Publ. Espec. Inst. Esp. Oceanogr., v. 24, p. 43-52, 1997b.

SEI, Superintendência de Estudos Econômicos e Sociais da Bahia. Balanço Hídrico do Estado da Bahia. Salvador: SEI, 1999. 249 p.

SILVA, V. R. F. Estudo sobre a comunidade ictioplanctônica da corrente do Brasil, entre Salvador, BA e Aracaju, SE. Salvador, 1997, 60f. Monografia (Bacharelado), Universidade Federal da Bahia.

SINQUE, C.; MUELBERT, J. H. Ichthyoplankton. In: SEELIGER, U.; ODEBRECHT, C.; CASTELLO, J. P. Subtropical Convergence Environments: The Coast and Sea in Southwestern Atlantic. Hamburg: Springer- Verlag, 1997. p. 51-54.

SMITH, P. E.; RICHARDSON, S. L. Standart techniques for pelagic fish eggs and larvae surveys. FAO Fish. Tech. Pap., v. 175, p. 1-100, 1977.

STRICKLAND, J. D.; PARSONS, T. R. A manual of seawater analysis. Bull. Fish. Res. Board Can, v. 125, p. 1-205, 1965.

SOUZA, F. B. C. Distribuição e abundância de larvas da família Scaridae, na Zona Econômica Exclusiva, entre Recife e Salvador, durante o REVIZEE Nordeste II. Salvador, 1999, $26 f$. Monografia (Bacharelado), Universidade Federal da Bahia.

VELAME, M. P. Composição, Distribuição e Abundância do Ictioplâncton nos Bancos Oceânicos da Cadeia Norte e Arquipélagos de Fernando de Noronha e de São Pedro e São Paulo. Salvador, 2001, 52f. Monografia (Bacharelado), Universidade Federal da Bahia.

ZAR, J. H. Bioestatistical Analysis. Prentice Hall, New York, 1984. 
Apêndice 1 - Lista dos taxa identificados na costa norte da Bahia, Brasil.

FILO CHORDATA

Divisão Teleostei

Subdivisão Elopomorpha

Ordem Anguilliformes

Subordem Congroidei

Família Ophichthidae

Sub-família Ophichthinae

Ophichthus sp

Subdivisão Clupeomorpha

Ordem Clupeiformes

Subordem Clupeoidei

Família Engraulidae

Família Clupeidae

Harengula clupeola (Cuvier,1829)

Opisthonema oglinum (Le Sueur,1818)

Subdivisão Euteleostei

Superordem Stenopterygii

Ordem Stomiiformes

Família Gonostomatidae

Sub-família Gonostomatinae

Cyclothone sp.

Superordem Cyclosquamata

Ordem Aulopiformes

Subordem Alepisauroidei

Família Synodontidae

Sub-família Synodontinae

Synodus foetens (Linnaeus,1766)

Superordem Scopelomorpha

Ordem Myctophiformes

Família Myctophidae

Sub-família Myctophinae

Benthosema sp.

Sub-família Lampanichtinae

Ceratoscopelus sp.

Diaphus sp.

Lampadena sp.

Superordem Paracanthopterygii

Ordem Ophidiiformes

Subordem Ophidioidei

Família Ophidiidae

Sub-família Ophidiinae

Lepophidium sp.

Ordem Gadiiformes

Família Macrouridae

Família Bregmacerotidae

Bregmaceros sp.

Continua... 
Subordem Scombroidei

Família Scombridae

Sub-família Scombrinae

Scomberomorus cavalla (Cuvier,1829)

Auxis thazard (Lacépède,1803)

Subordem Stromateoidei

Família Stromateidae

Ordem Pleuronectiformes

Peprilus sp.

Subordem Pleuronectoidei

Família Bothidae

Bothus ocellatus (Agassiz,1831)

Syacium micrurum Ranzani,1840

Família Paralichthidae

Família Soleidae

Citharichthys spilopterus Gunther,1862

Achirus lineatus (Linnaeus,1758)

Família Cynoglossidae

Symphurus plagusia (Bloch \& Schneider,1801)

Ordem Tetraodontiformes

Subordem Tetraodontoidei

Família Balistidae

Balistes vetula Linnaeus, 1758

Família Monacanthidae

Família Tetraodontidae

Sub-família Tetraodontinae

Lagocephalus laevigatus (Linnaeus,1766) 